Original Research Paper

\title{
Selenium Enriched Peanut Protein Alleviates Alcohol-Induced Liver Damage in Mice by Modulating the Composition of the Gut Microbiota
}

\author{
${ }^{1,3}$ Lin Gao, ${ }^{1}$ Jiawei Yuan, ${ }^{1}$ Yuhuan Cheng, ${ }^{1}$ Mengling Chen and ${ }^{2,3}$ Jihong Wu \\ ${ }^{1}$ School of Biology and Food Engineering, Changshu Institute of Technology, Changshu 215500, China \\ ${ }^{2}$ College of Food Science and Nutritional Engineering, China Agricultural University, Beijing 100083, China \\ ${ }^{3}$ National Engineering Research Center for Fruits and Vegetables Processing, \\ Ministry of Science and Technology, Beijing 100083, China
}

\author{
Article history \\ Received: 02-09-2021 \\ Revised: 01-11-2021 \\ Accepted: 04-11-2021 \\ Corresponding Author: \\ Jihong Wu \\ China Agricultural University, \\ No. 17, Qinghua East Road, \\ Haidian District, Beijing \\ 100083, China. \\ E-mail: wjhcau@ hotmail.com
}

\begin{abstract}
Alcohol Use Disorder (AUD) is one of leading external causes of mortality. Selenium-enriched Peanut Protein (SePP) is a type of Se compound present in Se-enriched plants. Our previous studies showed that SePP possesses potential therapeutic properties and could alleviate alcoholinduced AML-12 cytotoxicity. It spurred us to determine the underlying mechanisms and roles of SePP on gut microbiota. Here, ICR mice were fed either standard diet or ethanol $(30 \%, \mathrm{v} / \mathrm{v}, 10 \mathrm{~mL} / \mathrm{kg}$ bw/day) intragastric administration for 8 weeks. The intervention groups were divided into different doses of SePP groups and Se compounds groups, like Selenomethionine (SeMet) group, sodium selenite group. The composition of the gut microbiota was investigated by analyzing 16S rRNA gene sequences. Body weight, lipid metabolism markers, serum insulin and oxidative stress were assessed. Treatment with SePP at a certain dosage $(25 \mu \mathrm{g} / \mathrm{kg}$ bw/day in Se) and SeMet reversed diversity loss and community alterations in the gut microbiota of the AUD group, as evidenced by an increased abundance of Firmicutes and decreased abundances of Bacteroidetes and Verrucomicrobia in AUD mice. SePP suppressed the relative abundance of the Rikenellaceae_RC9 gut group and increased those of Lachnospiraceae and Ruminococcaceae, which are associated with lipid metabolism and Short-Chain Fatty Acids (SCFAs) production. The study suggested that SePP has the potential to be used as a supplement for alleviating alcohol-induced liver damage.
\end{abstract}

Keywords: Selenium-Enriched Aeanut Protein (SePP), Selenomethionine (SeMet), Alcohol Use Disorder (AUD), Gut Microbiota

\section{Introduction}

Alcohol Use Disorder (AUD), also called alcohol addiction, is one of leading external causes of mortality (Satish, 2003). According to the World Health Organization (WHO), 6\% of all global deaths or 3.3 million deaths are closely related to alcohol (Bajaj, 2019). Alcohol use disorder can lead to alcoholic liver toxicity, which is the leading risk of inflammation, fibrosis, or sclerosis of the liver and may even lead to cancer if left unchecked (Bajaj, 2019). Early symptoms of AUD are probably liver damages, such as oxidative stress, lipid peroxidation and certain damage of the liver tissue (Nurmela et al., 2015).
The pathophysiological mechanisms of liver injury caused by alcohol still incompletely understood, which is always multifactorial (Stärkel and Schnabl, 2016). Among these factors, alcohol-induced immune responses, metabolic disturbance and inflammation seems to play a crucial role (Bajaj, 2019; Wang et al., 2020). The gut microbiota is comprised of diverse microbes that are crucial to the well-being of their host (Clemente $e t$ al., 2012). In recent years, the relationship between gut microbiota and human health has been gradually revealed (David et al., 2014; Le Chatelier et al., 2013). A previous study (Satish, 2003) indicated chronic alcohol consumption result imbalance of the intestinal microbiota by changing the permeability of the intestinal mucosa. 
Cellular metabolites, like endotoxin will enter the blood and activate cells to release free radicals and cytokines by binding to specific receptors of liver cells, which in turn promote the release of large amounts of inflammatory mediators and cause damage to liver cells. Stärkel et al. (2018; Fan et al., 2019; Hartmann et al., 2015). However, here are largely unknown which factors can shape alcohol-associated microbiome. Recent research has suggested that ethanol feeding reduces the abundance of the phylum Firmicutes in mice and increases the intestinal levels of Verrucomicrobia (Cicenia et al., 2014; Hartmann et al., 2013; Yan et al., 2011a). Hartmann P reviewed this can be explained by increasing gastric $\mathrm{pH}$, causing intestinal dysmotility, altering bile flow and immune response (Hartmann et al., 2015).

Selenium (Se), an essential trace element, has attracted scientific attention for its beneficial effects against antioxidant activity-related diseases such as cancer, cardiovascular disease and liver disease (Rayman, 2020; Durguti et al., 2020; Sunday, 2021). Selenium-enriched Peanut Protein (SePP), a type of food source Se, is obtained from Se-enriched peanuts, which take up part of the supplemented sodium selenite applied to the soil for incorporation into organically bound Se (Gao et al., 2017; Nkansah et al., 2019). Reported that organic selenium has higher bioavailability than inorganic selenium and 70$95 \%$ of selenium in general food can be absorbed and utilized. Cereals and meat are considered to be important food sources of selenium, as selenium in these foods is mainly in the form of Se Cys and SeMet (Lavu et al., 2016). SeMet that selenomethionine replaced methionine with non-specific synthetic protein, which was more effective in increasing the body's selenium level and it has been reported to have a high absorption and utilization rate (Hinojosa Reyes et al., 2006). suggested the selenium in some plants is in the form of protein is more easy to be absorbed. Several studies have shown that Se compounds include inorganic Se like selenate and selenite and organic Se, such as Selenomethionine (SeMet), Selenocystine (SeCys) and so on, have an effect on gut microbiological compositions (Zhai et al., 2018; Gao et al., 2020; Cheng et al., 2021). Se-enriched foods perceived as contributing to the abundance of probiotic bacteria (Maseko et al., 2014; Zhu et al., 2019). Our previous studies showed that SePP possesses potential therapeutic properties and could alleviate alcohol-induced AML-12 cytotoxicity. It spurred us to determine the underlying mechanisms and roles of Se compounds on gut microbiota. In this study, the effects of supplementation with SePP on the gut microbiota in alcohol-treated mice and the influences on lipid metabolism, insulin resistance and oxidative stress were to evaluate.

\section{Materials and Methods}

\section{Nomenclature}

\section{Nomenclature was Followed}

Selenium, Se; Selenium enriched peanut, SePP; Alcohol use disorder, AUD; Selenomethionine, SeMet; Sodium selenite, NaSeO; Control group, N; Light doses of SePP group, SePPL; Middle doses of SePP group, SePPM; High doses of SePP group, SePPH; Short-chain fatty acids, SCFAs; Selenocystine, SeCys; Serum lipopolysaccharide, LPS; Alanine transaminase, ALT; Aspartate transaminase, AST; Serum lactate dehydrogenase, LDH; Serum total triglycerides, TG; Serum total cholesterol, CHO; Glutathione peroxidase, GSH-Px; Principal coordinate analysis, PCoA; Principal component analysis, PCA.

\section{Animal Experiment}

\section{Animal Experiment 1}

All animal experiments were carried out in accordance with the National Research Council Guidelines. Sixweek-old male ICR mice ( $\mathrm{n}=30$, Beijing, China) with four animals per cage, were maintained and provided with standard diet. The mice were acclimatized to the new environment for a week and then were randomly assigned to 5 groups for 8 weeks experiment: The normal control group $(N, n=6)$, AUD group (AUD, $n=6)$, SePP group (SePP, $25 \mu \mathrm{g} / \mathrm{kg} \mathrm{bw/day} \mathrm{in} \mathrm{Se,} \mathrm{by} \mathrm{intragastric}$ administration, $\mathrm{n}=6$ ), SeMet group (SeMet, $25 \mu \mathrm{g} / \mathrm{kg}$ bw/day in Se, by intragastric administration, $\mathrm{n}=6$ ) and sodium selenite group ( $\mathrm{NaSeO}, 25 \mu \mathrm{g} / \mathrm{kg}$ bw/day in $\mathrm{Se}$, by intragastric administration, $\mathrm{n}=6$ ). The administered dose was calculated in accordance with the current dietary reference intakes for human adults. All groups except for the $\mathrm{N}$ group received ethanol $(30 \%, \mathrm{v} / \mathrm{v})$ by gavage $(10 \mathrm{~mL} / \mathrm{kg}$ bw/day). The body weights of all animals were recorded weekly prior to intragastric administration. Feces were collected weekly and stored at $-80{ }^{\circ} \mathrm{C}$. At the end of the study, the mice were sacrificed after $12 \mathrm{~h}$ of fasting and the plasma and livers were collected.

\section{Animal Experiment 2}

Six weeks old ICR male mice ( $\mathrm{n}=24$, Beijing, China) were acclimatized to the environment for one week and then were randomly divided into 5 groups following: The AUD group (AUD, $n=6$ ), SePPL group (SePP, $6.25 \mu \mathrm{g} / \mathrm{kg} \mathrm{bw/day} \mathrm{in} \mathrm{Se,} \mathrm{by} \mathrm{intragastric}$ administration, $\mathrm{n}=6$ ), SePPM group (SePP, $25 \mu \mathrm{g} / \mathrm{kg}$ bw/day in Se, by intragastric administration, $n=6$ ) and $\mathrm{SePPH}$ group (SePP, $50 \mu \mathrm{g} / \mathrm{kg} \mathrm{bw/day} \mathrm{in} \mathrm{Se,} \mathrm{by}$ intragastric administration, $n=6)$. All groups received ethanol $(30 \%, \mathrm{v} / \mathrm{v})$ by gavage $(10 \mathrm{~mL} / \mathrm{kg} \mathrm{bw} /$ day $)$. Eight weeks later, animals were fasted and the plasma, liver and feces were collected. 


\section{Biochemical Analysis}

Serum Lipopolysaccharide (LPS), such as ALT, AST, LDH, TG and CHO quantification was measured by a blood biochemistry analyzer 7020 (Hitachi, Tokyo, Japan). The mouse insulin Enzyme-Linked Immunosorbent Assay (ELISA) kit (ALPCO, US) was used for plasma GSH-Px activities and serum insulin quantitative.

\section{Gut Microbiota Analysis}

It was a modification of that in a previous report (Xu et al., 2020). The E.Z.N.A. ${ }^{\circledR}$ Stool DNA Kit (Omega Bio-tek, Norcross, GA, U.S.) was used for extracting total genome DNA from mice faeces, following the manufacturer's instructions. Using primers $338 \mathrm{~F}$ and 806R, the V4-V5 region of the bacterial 16S ribosomal RNA gene was amplified by PCR, where the barcode is the unique 8 base sequence of each sample. Each PCR product was visualized on a $2 \%$ agarose gel and purified using the AxyPrep DNA Gel Extraction Kit and quantified using Quanti Fluor ${ }^{\mathrm{TM}}$-ST (Promega, U.S.). Purified amplicons were pooled in equimolar and pairedend sequenced $(2 \times 250)$ on an Illumina MiSeq platform. The taxonomy of each 16S rRNA gene sequence was analyzed by RDP Classifier (http://rdp.cme.msu.edu/) against the silva (SSU115)16S rRNA database using confidence threshold of 70\% (Amato et al., 2013).

\section{Statistical Analysis}

SPSS software version 20 (IBM Corporation, NY, USA) was performed for statistical analyses. All data are expressed as mean \pm Square Deviation (SD). Duncan's multiple range tests were implemented to identify statistical separation among the means. Origin Pro version 9.0 and Graph Pad Prism version 7 were used for data plotting.

\section{Results}

\section{Effects of Different Se Compounds on the Gut Microbiota Momposition in AUD Mice}

The mice body weight gains in the $\mathrm{N}$ groups were 6.43 $\pm 1.01 \mathrm{~g}$ and in the AUD groups were $3.47 \pm 0.79 \mathrm{~g}$, after eight weeks-continuous treatment with $10 \mathrm{~mL} / \mathrm{kg}$ bw/day ethanol $(30 \%, v / v)$. After the interventions with $\mathrm{Se}$ compounds such as SePP, selenomethionine and sodium selenite with the same selenium concentration ( $25 \mu \mathrm{g} \mathrm{Se} / \mathrm{kg}$ bw/day) ( $p<0.01$, Fig. 1A), the body weights of the AUD mice recovered significantly. AUD administration increased the liver weight index, which was significantly decreased after the intragastric administration of Se compounds ( $p<0.01$, Fig. 1B).

Multivariate analysis was used to compare the gut microbiota composition in the five experimental groups, such as the N, AUD, SePP, SeMet and $\mathrm{NaSeO}$ groups, at phylum and genus levels. Principal Coordinate Analysis $(\mathrm{PCoA})$ revealed that the intestinal microbial communities were altered by alcohol and the Se compounds (Fig. 2A). The boxplot in Fig. 2A represents the discrete distributions of different groups on the PC1 axis and shows that when the mice were intragastrically treated with the same concentration of Se, the SePP group showed the furthest distance from the AUD group, followed by the SeMet group and then the $\mathrm{NaSeO}$ group (Fig. 2A). The relative abundance of the predominant taxa identified from sequencing in the five groups was compared to reveal the specific changes in the gut microbiota, as shown in Fig. 2B. At the phylum level, Bacteroidetes and Firmicutes were the dominant phyla in all groups, representing more than $90 \%$ of the relative abundances. Among the dominant phyla. After alcohol treatment, the relative abundance of Firmicutes was decreased, whereas there was an increase in Verrucomibrobia (Fig. 2B). Pretreatment with SePP and $\mathrm{NaSeO}$ markedly inhibited ethanol-induced alterations in the relative abundance of Verrucomibrobia $(p<0.01)$. SePP treatment also significantly restored the relative abundance of Firmicutes $(p<0.05)$. However, treatment with inorganic selenium did not ameliorate the changes in abundance induced by alcohol or even had a more significant tendency. Then a genus-level analysis was performed to further explore the differences (Fig. 2C). It turned out that excessive alcohol compared with the $\mathrm{N}$ condition significantly increased the relative abundance of Rikenellaceae_RC9_gut_group belonging to the Bacteroidetes phylum and decreased significantly the relative abundance of uncultured_f_Ruminococcaceae belonging to the Firmicutes phylum. Treatment with SePP or SeMet suppressed the changes, but not $\mathrm{NaSeO}$.

\section{SePP Improves Lipid Metabolism and Antioxidant Intervention in AUD Mice}

Liver disease is characterized by enhanced lipogenesis and oxidative stress. Here, we found that supplementation with different doses of SePP decreased the levels of serum lipids significantly, including ALT, AST, TG and CHO $(p<0.05)$, in AUD mice (Fig. 3A-E), with SePPM and $\mathrm{SePPH}$ performing better than SePPL. The GSH-Px levels in the SePP group were significantly increased in comparison with those in the AUD group (Fig. 3F), which suggested that intervention with SePPs, including SePPL, SePPM and $\mathrm{SePPH}$, alleviated AUD-induced oxidative stress. The serum insulin levels in the SePP groups were decreased significantly $(p<0.05)$ compared with those in the AUD groups (Fig. 3G), which suggested that intervention with SePP could influence insulin resistance in AUD mice. 
A

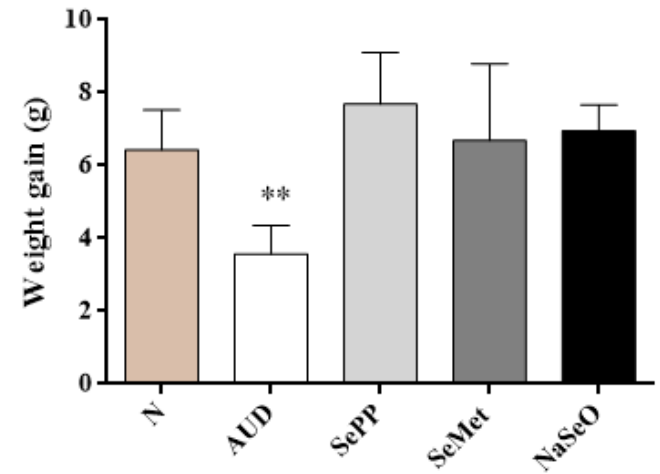

B

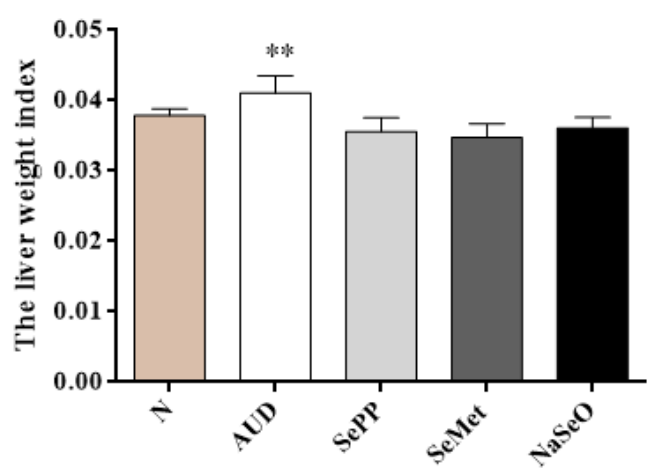

Fig. 1: SePP ameliorated the body weight gain and reduced the liver weight index in AUD mice. Body weight gain (A) and liver weight index (B). Values are expressed as the mean $\pm \mathrm{SD},{ }^{*} p<0.05,{ }^{* *} p<0.01$
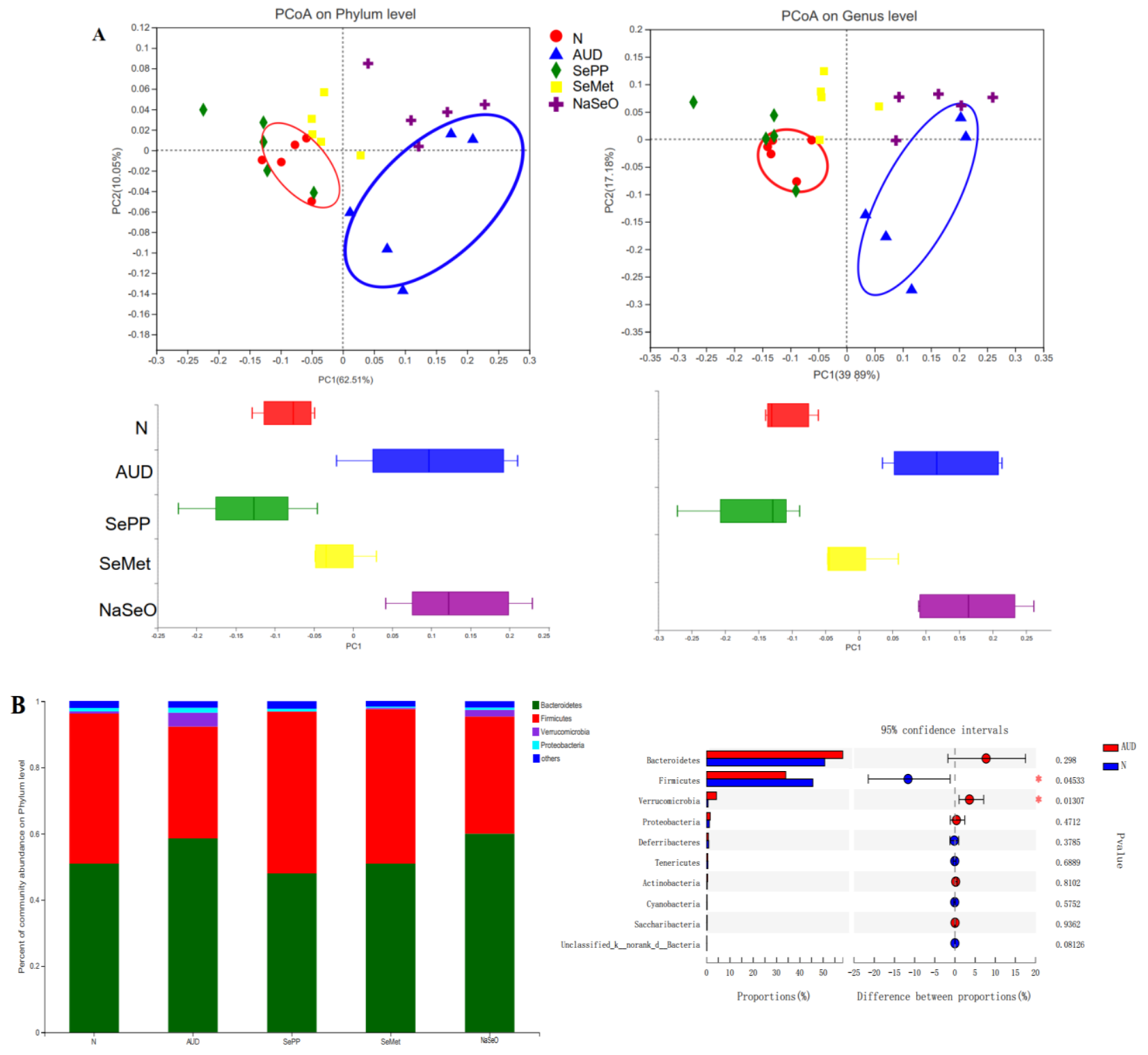

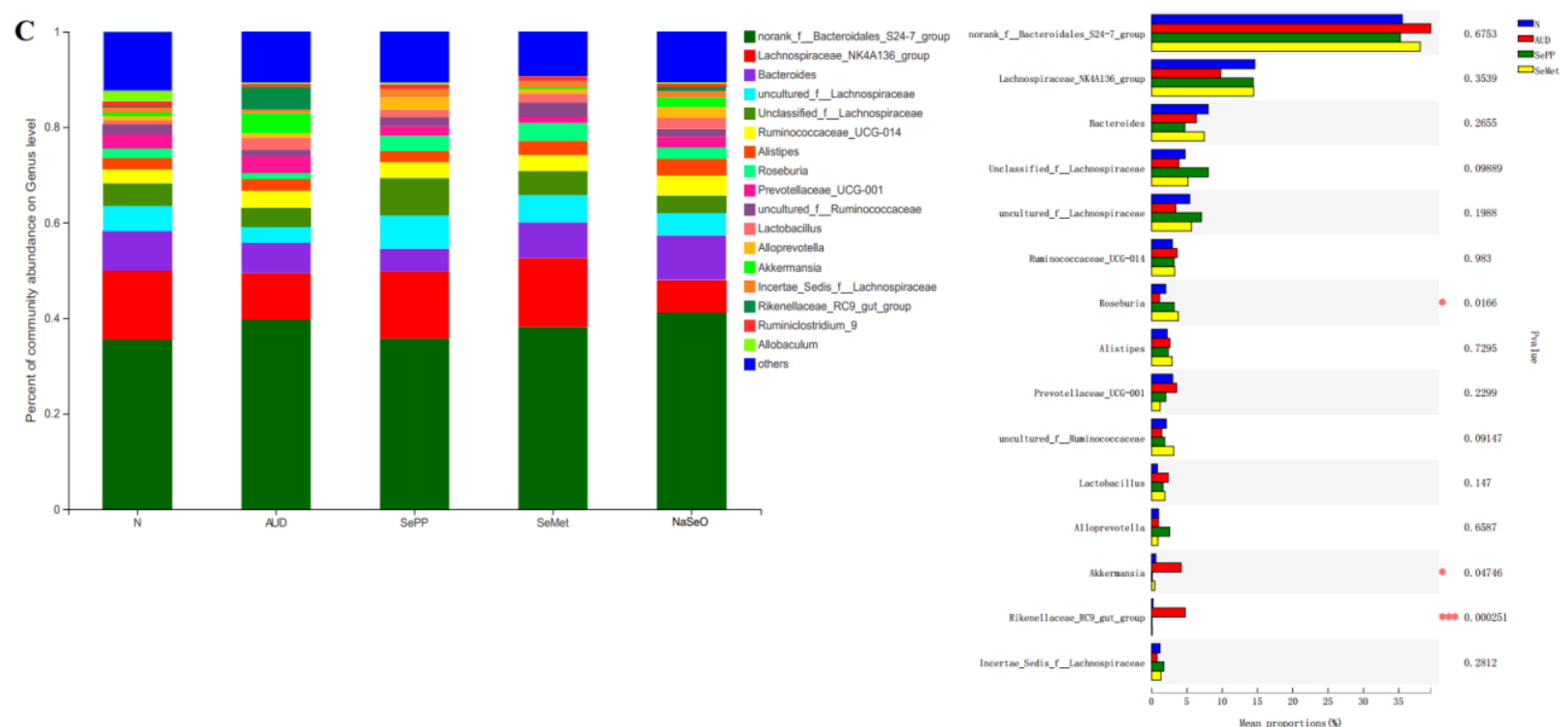

Fig. 2: Effects of different Se species on the gut microbiota composition in AUD mice. Principal Coordinate Analysis (PCoA) at the phylum and genus levels (A), gut microbiota composition at the phylum level (B) and gut microbiota composition at the genus level (C). The data are expressed as the mean \pm SD
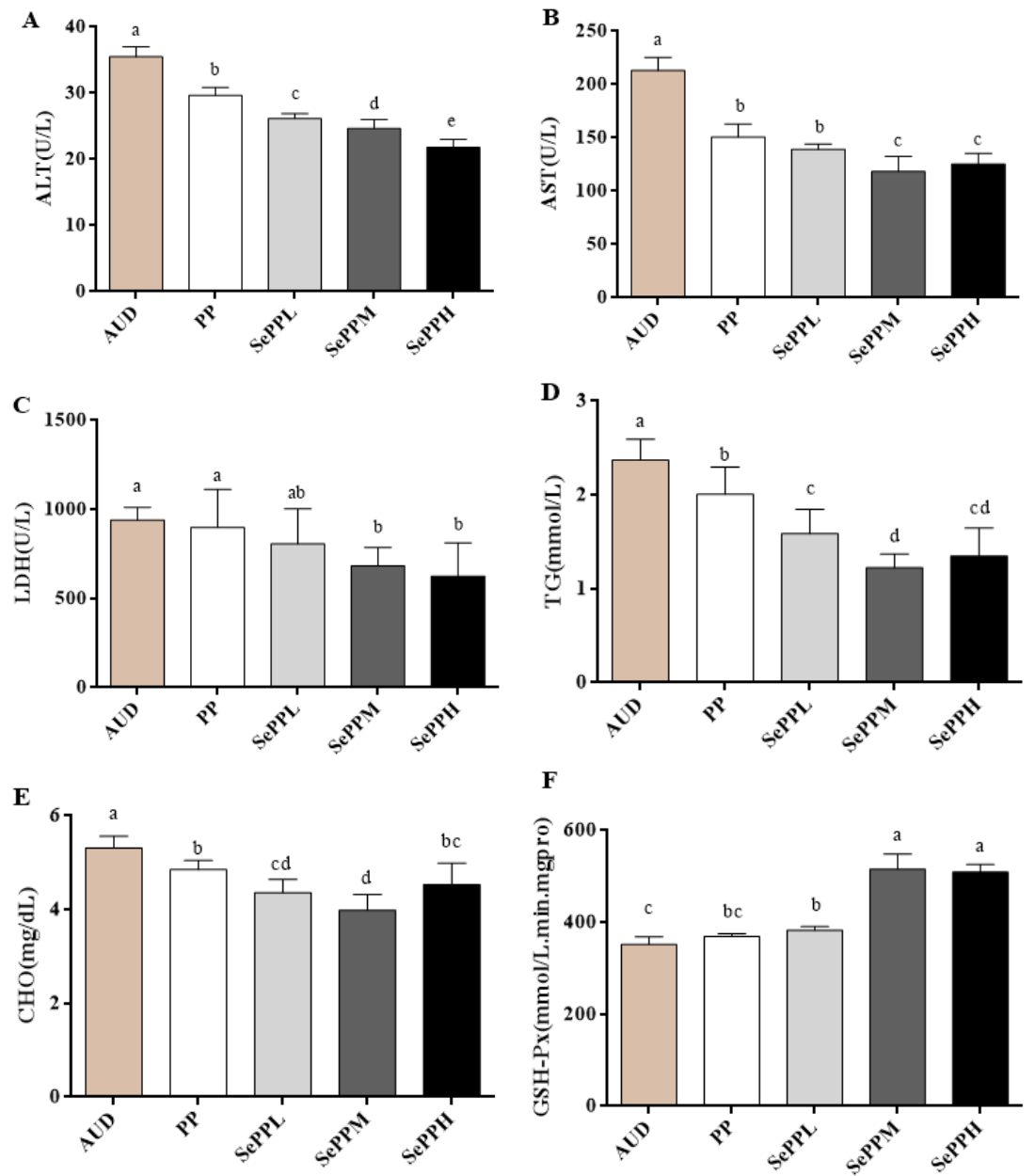


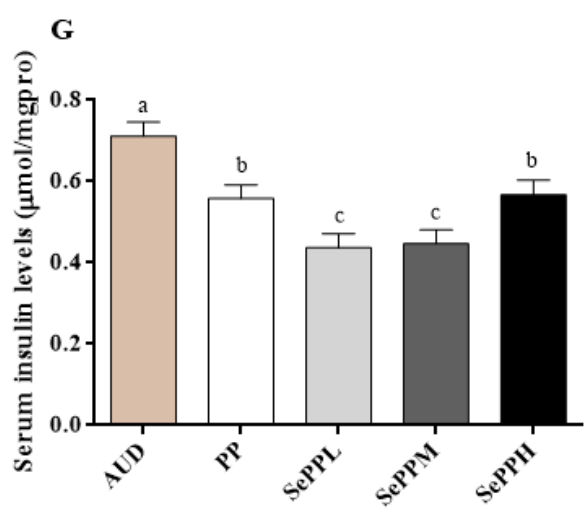

Fig. 3: SePP improves lipid metabolism and antioxidant intervention in AUD mice. The Levels of Alanine Transaminase (ALT) (A), Aspartate Transaminase (AST) (B), serum Lactate Dehydrogenase (LDH) (C), serum total Triglycerides (TG) (D), serum total Cholesterol (CHO) (E) Glutathione Peroxidase (GSH-Px) (F) and serum insulin (G) are shown. The data are expressed as the mean $\pm \mathrm{SD}(\mathrm{n}=6)$. The different letters denote statistically significant differences between the groups $(p<0.05)$
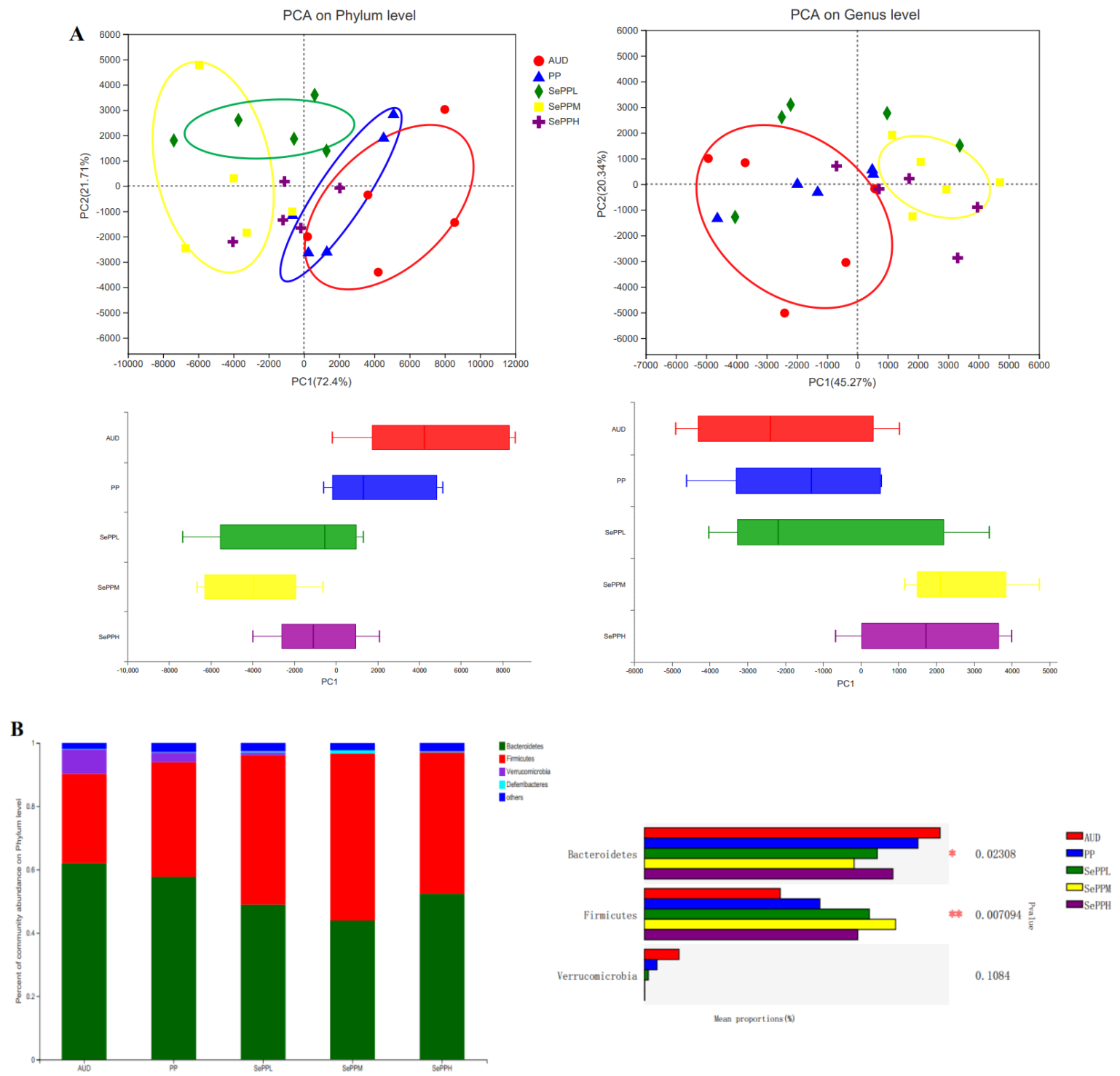

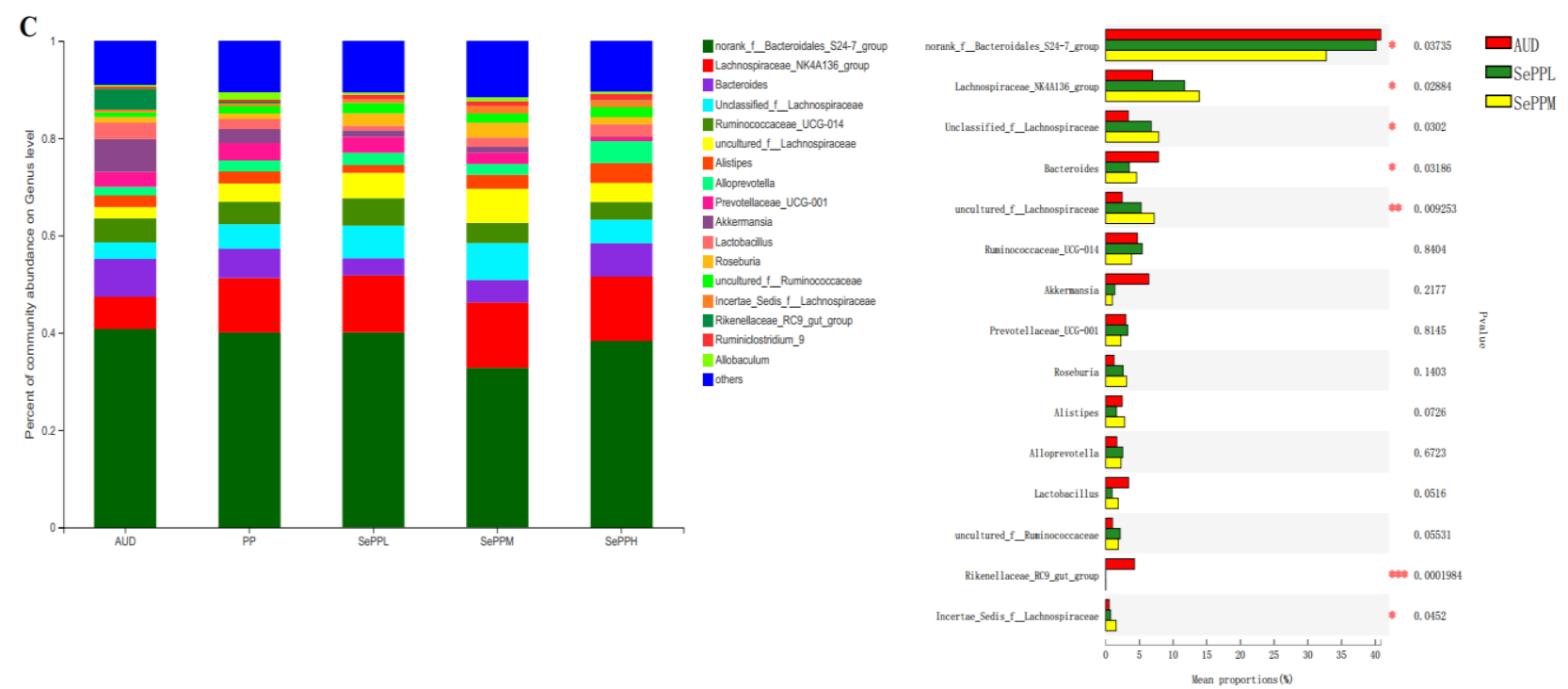

Fig. 4: Effects of different SePP doses on the gut microbiota composition in AUD mice. Principal Component Analysis (PCA) at the phylum and genus levels (A), gut microbiota composition at the phylum level (B) and gut microbiota composition at the genus level (C). The data are expressed as the mean $\pm \mathrm{SD}$

\section{Effects of SePP on Gut Microbiota Composition in AUD Mice}

Multivariate analysis of variance of Principal Component Analysis (PCA) matrix scores indicated a statistically significant separation between the microbiota of the AUD and SePPM groups (Fig. 4A). The boxplot in Fig. 4A represents the discrete distributions of different groups on the PC1 axis and shows that the SePPM group showed the furthest distance from the AUD group, followed by the SePPM and SePPL groups and then the PP group (Fig. 4A).

At the phylum level, supplementation with PP, SePPL and SePPM markedly increased the relative abundance of Firmicutes $(p<0.01)$ and inhibited the relative abundance of Bacteroidetes $(p<0.05)$, compared with the AUD group. The SePPM group performed best. The SePPL treatment was better than the PP treatment. It showed that treatment with appropriate concentration of SePP could relieve alcoholic liver injury while treatment with excessive SePP may cause toxicity.

Detailed analysis at the genus level indicated that the abundances of Rikenellaceae_RC9 gut_group and Bacteroides, which were found to be enhanced by alcohol, were reversed by SePP. Notably, in comparison with AUD mice, SePP enhanced a variety of Lachnospiraceae species that negatively correlated with liver disease and had Se dose-dependent effects at a certain dosage $(25 \mu \mathrm{g} / \mathrm{kg}$ bw/day in $\mathrm{Se})$.

\section{Correlation Analysis}

To investigate the underlying mechanisms and roles of Se compounds in regulating gut microbiota and parameters associated with lipid metabolism, Pearson correlations analysis shown in Fig. 5 was performed to reveal possible correlation between gut microbiota and metabolic parameters, such as serum biochemicals (ALT, AST, LDH, CHO, TG), GSH-Px and insulin content (Fig. 5A). Gut microbiota like Firmicutes, Bacteroidetes, Verrucomicrobia, Rikenellaceae_RC9, Lachnospiraceae and Ruminococcaceae, were paid much more attention. Firmicutes and Ruminiclostridium_9 (ALT, AST, TG, $p<0.001$ ), Incertae_Sedis_f_Lachnospiraceae (ALT, LDH, TG, $p<0.01)$ were negatively correlated with serum biochemical, whereas Verrucomicrobia (ALT, $p<0.01$, AST, LDH, $p<0.05)$, Rikenellaceae_RC9 gut_group (ALT, AST, TG, $p<0.001$ ) and were positively correlated with serum biochemicals. Firmicutes $(p<0.05), \quad$ Ruminiclostridium_9 belonging to the Firmicutes phylum $(p<0.001)$ and Lachnospiraceae $(p<0.001)$, were positively correlated with GSH-Px. Firmicutes $(p<0.001)$, Ruminiclostridium_9 $(p<0.001)$, Bacteroides $(p<0.001)$ were positively correlated with insulin content and Verrucomicrobia $(p<0.05)$ were negatively correlated with insulin content. 
A

Pearson Correlation Heatmap

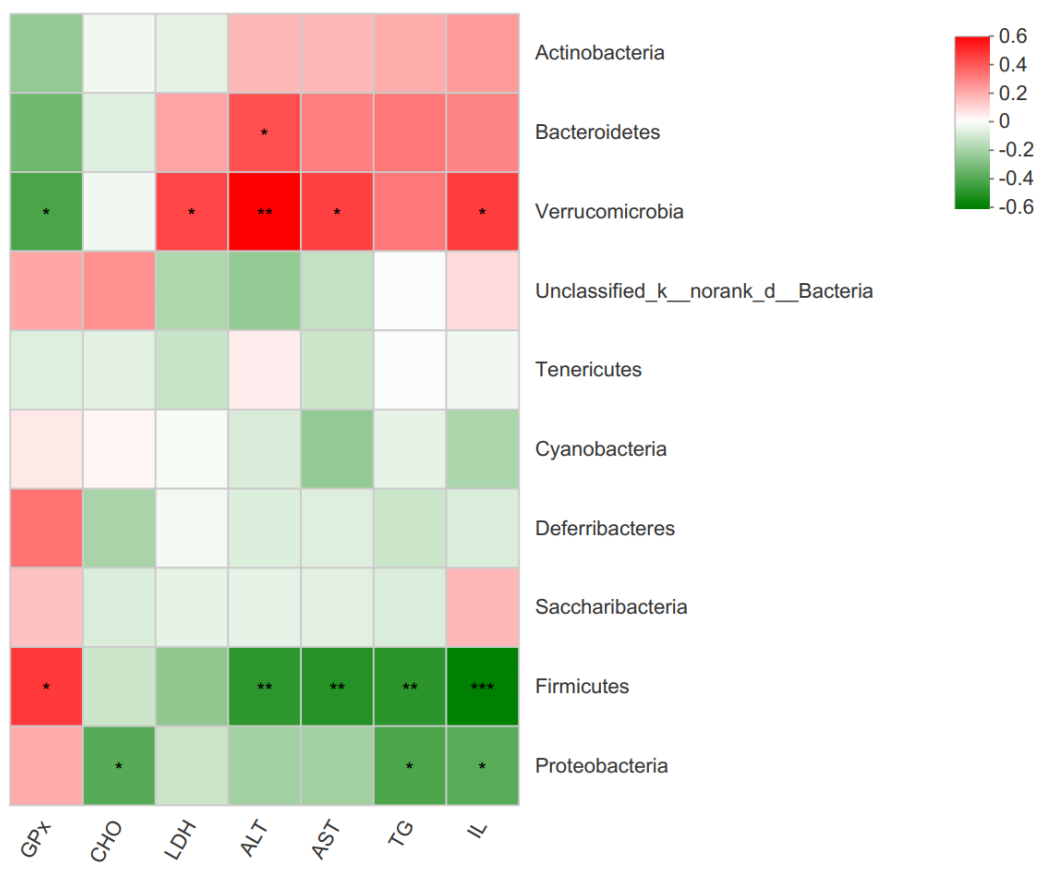

Pearson Correlation Heatmap

B

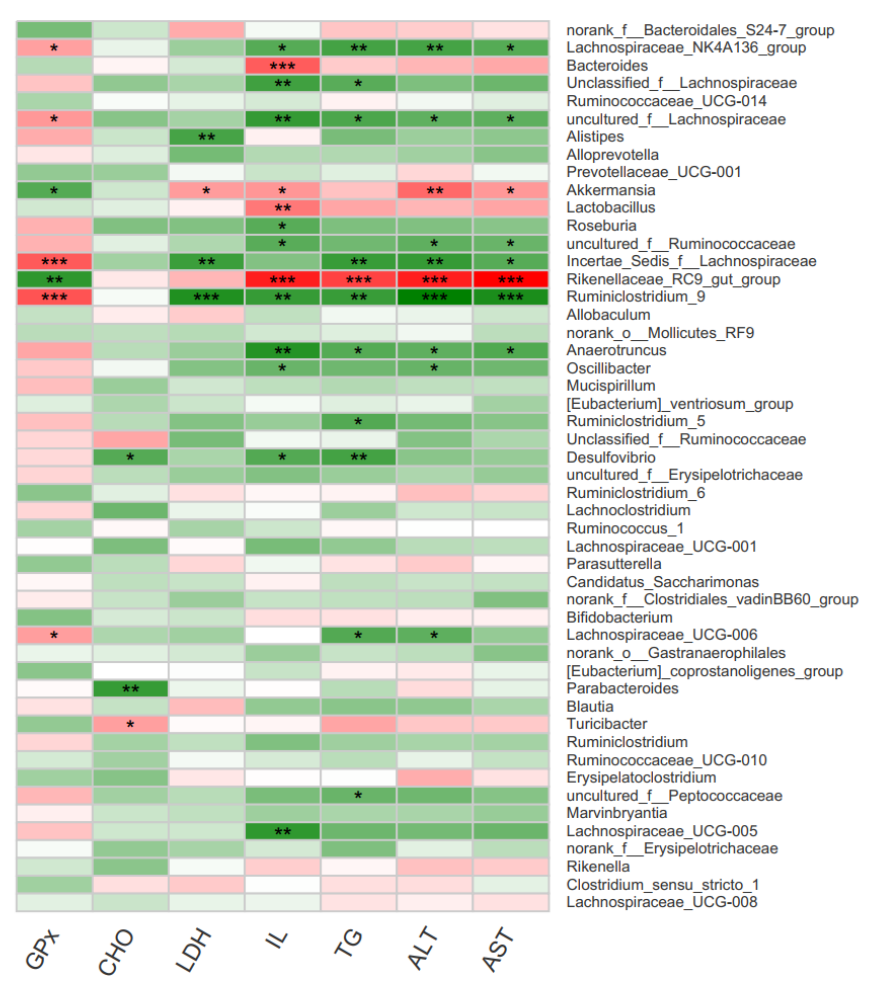

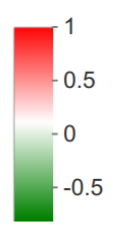

$-0.5$

Fig. 5: Pearson correlation heat map between the abundances of gut microbiota and metabolic parameters of the host. Pearson correlation heat map at the phylum level (A) and Pearson correlation heatmap at the genus level (B). The data are expressed as the mean $\pm \operatorname{SD}(n=6)$ 


\section{Discussion}

Liver disease caused by excessive drinking is a major problem for global public health (Sugimoto and Takei, 2017; Zhao et al., 2018). The aggravation of liver disease is concerned with several issues, such as comorbidities, nutritional status and the amount and duration of alcohol consumption (Louvet and Mathurin, 2015). Among these factors, alcohol-related changes of the intestinal microbiota and its metabolites in liver pathophysiology to be highlighted, recently (Bajaj, 2019; Stärkel and Schnabl, 2016). Selenium (Se), an essential trace element, has attracted scientific attention for its beneficial effects against antioxidant activity-related diseases such as cancer, cardiovascular disease and liver disease (Roman et al., 2014; Gao et al., 2017; Zeng and Combs, 2008). Our previous studies showed that SePP could alleviate alcohol-induced AML-12 cytotoxicity. The present research has proven that human health and disease are related to the intestinal flora. The possible reason may be that the diversity and the composition of the gut microbiota plays a major role by shaping human metabolism and immune system (Suez et al., 2014; Milosevic et al., 2019). The current research has proved that human health and diseases. The possible reason may be that the diversity and composition of the intestinal flora play an important role in shaping our immune system and metabolism. Some studies also reported that excessive drinking can cause changes in the composition of the intestinal flora, mucosal inflammation and intestinal barrier disorders (Wang et al., 2020; Meroni et al., 2019). Whether SePP exerts beneficial effects in alcohol-treated mice and how it changes the gut microbiota remains unknown.

We aimed to study the effects of supplementation with SePP on the gut microbiota of alcohol-treated mice in two ways. First, we revealed that supplementation with different Se compounds, such as SePP, selenomethionine and sodium selenite, with the same selenium concentration in AUD mice prevented increase in their weight gain and the liver weight index.

Then, we focused on their alteration of the gut microbiota composition. Second, different doses of SePP regulated lipid metabolism, insulin resistance and oxidative stress and SePPM and SePPH were better treatments than SePPL. Then, SePP at different doses was utilized to further explore the roles of the gut microbiota.

It showed that treatment with appropriate concentration of SePP $(25 \mu \mathrm{g} / \mathrm{kg}$ bw/day in Se $)$ could relieve alcoholic liver injury while treatment with excessive SePP may cause toxicity. The range between the dietary requirement and the safe intake of selenium is narrow. According to various studies, when the intake of selenium is less than $30 \mu \mathrm{g}$ per day, the intake of selenium is insufficient and when the daily intake is higher than $900 \mu \mathrm{g}$, there will be a certain security threat to the body (Brigelius-Flohé, 2018). The risk of alcohol-induced liver disease increases when selenium is low in foods, since drinking alcohol may reduce the intake of food and this also easier to promote selenium deficiency (Navarro-Alarcon and Cabrera-Vique, 2008).

Studies have shown that the treatment of Se-enriched C. hupingshanensis aqueous extract has a positive effect on the intestinal flora, intestinal redox status and the production of short-chain fatty acids in rats (Cheng et al., 2021; Umana et al., 2020). Se-containing green tea (Zhu et al., 2019) or Se-enriched Bifidobacterium longum DD98 (Kousha and Yeganeh, 2019) supplementation effectively increased the abundance of lactic acid bacteria, while MeSeCys, SeCys2 and SeMet supplementation attained the same effect. As a result of this study, SePP and SeMet supplementation affects the gut microbiota, as evidenced by the decreased abundance of Bacteroidetes and Verrucomicrobia and the increased abundance of Firmicutes in AUD mice. Alcohol consumption is associated with quantitative and qualitative changes in the intestinal microbiota, also called intestinal dysbiosis. Several studies have shown that alcohol-treated mice show higher intestinal levels of Bacteroidetes and a lower abundance of Firmicutes (Fan et al., 2019; Hartmann et al., 2015; Chen et al., 2015b). There is evidence that alcoholtreated mice show higher intestinal levels of Verrucomicrobia (Yan et al., 2011a; Hartmann et al., 2013; Yan et al., 2011b). Another study reported similar findings, which showing a reduction in Firmicutes and conversely an enhanced abundance of Bacteroidetes and Verrucomicrobia in mice gut microflora after excessive alcohol intake (Yan et al., 2011b). More detailed analysis in these mice demonstrated that this is related to the downregulation of gene and protein expression of bactericidal c-type lectins Reg3b and Reg3g in the small intestine caused by alcohol (Yan et al., 2011b). These changes confirmed gut microbiota composition play a role in alcohol-induced damage both in the liver and in the intestine, which attributes to intestinal barrier integrity alteration and proinflammatory mediators into the portal circulation release (Stärkel et al., 2018).

Based on this, further observation was performed at the genus level that supplement with SePP at certain dosage $(25 \mu \mathrm{g} / \mathrm{kg}$ bw/day in $\mathrm{Se})$ suppressed the relative abundance of the Rikenellaceae_RC9 gut_group. A previous study showed that the undefined genera of the Rikenellaceae family is positively correlated with liver triglyceride content (Welly et al., 2016), which was consistent with our results that the Rikenellaceae_RC9 gut_group was positively correlated with ALT, AST and TG. ALT, AST and TG, inflammatory markers of liver disease, are associated with excessive alcohol consumption and are negatively correlated with intestinal butyrate (Cresci et al., 2014; Cresci et al., 2017; Rivière et al., 2016).

In addition, compared with AUD, SePP can significantly increase the relative abundances of unclassified Lachnospiraceae, Ruminococcaceae and 
several other genera, which have been reported to be negatively associated with liver injury (Chen et al., 2015a). Studies have also shown that alcohol increased the abundance of Bacteroidetes and reduced the abundance of Lactobacillus species, Ruminococcus genus and other flora related to the production of short-chain fatty acids (Fan et al., 2019). Ruminococcaceae and Lachnospiraceae, belonging to Firmicutes, are widely believed to produce SCFAs that are important for the amelioration of chronic inflammatory diseases and the promotion of colonocyte health (Stärkel et al., 2018; Milosevic et al., 2019; Bajaj and Khoruts, 2020).

The microbiota can be modified in a relatively untargeted fashion with treatments such as antibiotics, prebiotics, probiotics, synbiotics and fecal microbiota transplantation (Stärkel and Schnabl, 2016; Louvet and Mathurin, 2015b; Chen et al., 2015b). Some studies have shown the mechanism of probiotic treatment and the fiber supplement has the effect of alleviating liver injury is improving intestinal barrier function. SCFAs dietary prevents alcoholic liver injury can be attributed to its effect of restoring the abundances of Bacteroidetes in the mouse intestine, which contributes to improving intestinal barrier function (Chen et al., 2015a; Cresci et al., 2014; Chen et al., 2015b). The results of this study show that supplement of SePP influenced the gut microbiota in AUD mice, which was associated with lipid metabolism and SCFA production.

\section{Conclusion}

Se-enriched foods have been shown to increase the abundance of beneficial bacteria. Whether SePP, a type of food source Se, is obtained from Se-enriched peanuts, exerts beneficial effects in alcohol-treated mice and how it influence the gut microbiota remains unknown. The effects of supplementation with SePP on the gut microbiota in alcohol-treated mice and the influences on lipid metabolism, insulin resistance and oxidative stress were evaluates. It turned out that SePP and its dominating selenium compound SeMet supplementation markedly changes the gut microbiota composition, as evidenced by the decreased abundances of Bacteroidetes and Verrucomicrobia and the increased abundance of Firmicutes in AUD mice. SePP at a certain dosage $(25 \mu \mathrm{g} / \mathrm{kg}$ bw/day in Se) suppressed the relative abundance of the Rikenellaceae_RC9 gut group and increased those of Lachnospiraceae and Ruminococcaceae, which are negatively correlated with ALT, AST and TG. ALT, AST and TG, inflammatory markers of liver disease and positively correlated with SCFA production, which are widely believed to be important for the amelioration of chronic inflammatory diseases and the promotion of colonocyte health. Previous studies suggest that SePP possesses potential therapeutic properties and could alleviate alcohol-induced AML-12 cytotoxicity.
Collectively, findings of this study will provide new insights into AUD prevention based on plant source Se. Our study is highly innovative as it is the first to focus on the effects of Se-enriched peanut extract and its dominating selenium compound on the gut microbiota in alcohol-treated mice. The gut-promoting function of specific chemical forms of Se in SePP will be identified in the further research.

\section{Funding Information}

This research was funded by Natural Science Foundation of Jiangsu Province (No. BK20181035).

\section{Author's Contributions}

Lin Gao: Designed and executed the experiments and wrote the manuscript.

Jiawei Yuan and Yuhuan Cheng: Analyzed the data and revised the manuscript.

Mengling Chen and Jihong Wu: Conceived and designed the experiments. All authors contributed to and have approved the final manuscript.

\section{Ethics}

This article is original and contains unpublished material. The corresponding author confirms that all of the other authors have read and approved the manuscript and no ethical issues involved.

\section{References}

Amato, K. R., Yeoman, C. J., Kent, A., Righini, N., Carbonero, F., Estrada, A., Rex Gaskins, H., Stumpf, R. M., Yildirim, S., Torralba, M., Gillis, M., Wilson, B. A., Nelson, K. E., White, B. A., \& Leigh, S. R. (2013). Habitat degradation impacts black howler monkey (Alouatta pigra) gastrointestinal microbiomes. ISME Journal. doi.org/10.1038/ismej.2013.16

Bajaj, J. S. (2019). Alcohol, liver disease and the gut microbiota. Nature Reviews Gastroenterology and Hepatology, 16(4), 235-246. doi.org/10.1038/s41575-018-0099-1

Bajaj, J. S., \& Khoruts, A. (2020). Microbiota changes and intestinal microbiota transplantation in liver diseases and cirrhosis. Journal of Hepatology, 72(5), 1003-1027. doi.org/10.1016/j.jhep.2020.01.017

Brigelius-Flohé, R. (2018). Selenium in human health and disease: An overview. Molecular and Integrative Toxicology, 14(7), 3-26. doi.org/10.1007/978-3-319-95390-8_1

Chen, P., Stärkel, P., Turner, J. R., Ho, S. B., \& Schnabl, B. (2015a). Dysbiosis-induced intestinal inflammation activates tumor necrosis factor receptor I and mediates alcoholic liver disease in mice. Hepatology. doi.org/10.1002/hep.27489 
Chen, P., Torralba, M., Tan, J., Embree, M., Zengler, K., Stärkel, P., Van Pijkeren, J. P., DePew, J., Loomba, R., Ho, S. B., Bajaj, J. S., Mutlu, E. A., Keshavarzian, A., Tsukamoto, H., Nelson, K. E., Fouts, D. E., \& Schnabl, B. (2015b). Supplementation of saturated long-chain fatty acids maintains intestinal eubiosis and reduces ethanol-induced liver injury in mice. Gastroenterology, 148(1), 203-214.e16. doi.org/10.1053/j.gastro.2014.09.014

Cheng, Y., Huang, Y., Liu, K., Pan, S., Qin, Z., Wu, T., \& Xu, X. (2021). Cardamine hupingshanensis aqueous extract improves intestinal redox status and gut microbiota in Se-deficient rats. Journal of the Science of Food and Agriculture, 101(3), 989-996. doi.org/10.1002/jsfa.10707

Cicenia, A., Scirocco, A., Carabotti, M., Pallotta, L., Marignani, M., \& Severi, C. (2014). Postbiotic activities of lactobacilli-derived factors. Journal of Clinical Gastroenterology. doi.org/10.1097/MCG.0000000000000231

Clemente, J. C., Ursell, L. K., Parfrey, L. W., \& Knight, R. (2012). The impact of the gut microbiota on human health: An integrative view. In Cell. doi.org/10.1016/j.cell.2012.01.035

Cresci, G. A., Bush, K., \& Nagy, L. E. (2014). Tributyrin supplementation protects mice from acute ethanolinduced gut injury. Alcoholism: Clinical and Experimental Research. doi.org/10.1111/acer.12428

Cresci, G. A., Glueck, B., McMullen, M. R., Xin, W., Allende, D., \& Nagy, L. E. (2017). Prophylactic tributyrin treatment mitigates chronic-binge ethanolinduced intestinal barrier and liver injury. Journal of Gastroenterology and Hepatology (Australia). doi.org/10.1111/jgh.13731

David, L. A., Maurice, C. F., Carmody, R. N., Gootenberg, D. B., Button, J. E., Wolfe, B. E., Ling, A. V., Devlin, A. S., Varma, Y., Fischbach, M. A., Biddinger, S. B., Dutton, R. J., \& Turnbaugh, P. J. (2014). Diet rapidly and reproducibly alters the human gut microbiome. Nature. doi.org/10.1038/nature12820

Durguti, V., Aliu, S., Laha, F., \& Feka, F. (2020). Determination of iron, copper and zinc in the wine by FAAS. Emerging Science Journal, 4(5), 411-417. doi.org/10.28991/esj-2020-01240

Fan, J., Wang, Y., You, Y., Ai, Z., Dai, W., Piao, C., Liu, J., \& Wang, Y. (2019). Fermented ginseng improved alcohol liver injury in association with changes in the gut microbiota of mice. Food and Function, 10(9), 5566-5573. doi.org/10.1039/c9fo01415b

Gao, L., Luo, D., Hu, X., \& Wu, J. (2017). Se in Se-enriched peanut and losses during peanut protein preparation. International Journal of Food Science and Technology, 52(3), 843-850. doi.org/10.1111/ijfs.13347
Gao, Y., Xu, Y., Ruan, J., \& Yin, J. (2020). Selenium affects the activity of black tea in preventing metabolic syndrome in high-fat diet-fed Sprague-Dawley rats. Journal of the Science of Food and Agriculture, 100(1), 225-234. doi.org/10.1002/jsfa.10027

Hartmann, P., Chen, P., Wang, H. J., Wang, L., Mccole, D. F., Brandl, K., Stärkel, P., Belzer, C., Hellerbrand, C., Tsukamoto, H., Ho, S. B., \& Schnabl, B. (2013). Deficiency of intestinal mucin-2 ameliorates experimental alcoholic liver disease in mice. Hepatology. doi.org/10.1002/hep.26321

Hartmann, P., Seebauer, C. T., \& Schnabl, B. (2015). Alcoholic liver disease: The gut microbiome and liver cross talk. Alcoholism: Clinical and Experimental Research, 39(5), 763-775. doi.org/10.1111/acer.12704

Hinojosa Reyes, L., Marchante-Gayón, J. M., García Alonso, J. I., \& Sanz-Medel, A. (2006). Application of isotope dilution analysis for the evaluation of extraction conditions in the determination of total selenium and selenomethionine in yeast-based nutritional supplements. Journal of Agricultural and Food Chemistry, 54(5), 1557-1563. doi.org/10.1021/jf0523768

Kousha, M., \& Yeganeh, S. (2019). Synergistic effect of sodium selenite and Pediococcus acidilactici on growth, intestinal bacterial counts, selenium bioavailability, hepatic enzymes and non - specific immune response in rainbow trout (Oncorhynchus mykiss). September 2018, 1-14. doi.org/10.1111/anu.12968

Lavu, R. V. S., Van De Wiele, T., Pratti, V. L., Tack, F., \& Du Laing, G. (2016). Selenium bioaccessibility in stomach, small intestine and colon: Comparison between pure Se compounds, Se-enriched food crops and food supplements. Food Chemistry, 197, 382-387. doi.org/10.1016/j.foodchem.2015.08.001

Le Chatelier, E., Nielsen, T., Qin, J., Prifti, E., Hildebrand, F., Falony, G., Almeida, M., Arumugam, M., Batto, J. M., Kennedy, S., Leonard, P., Li, J., Burgdorf, K., Grarup, N., Jørgensen, T., Brandslund, I., Nielsen, H. B., Juncker, A. S., Bertalan, M., ... Yamada, T. (2013). Richness of human gut microbiome correlates with metabolic markers. Nature. doi.org/10.1038/nature12506

Louvet, A., \& Mathurin, P. (2015). Alcoholic liver disease: Mechanisms of injury and targeted treatment. In Nature Reviews Gastroenterology and Hepatology. doi.org/10.1038/nrgastro.2015.35

Maseko, T., Dunshea, F. R., Howell, K., Cho, H. J., Rivera, L. R., Furness, J. B., \& Ng, K. (2014). Seleniumenriched Agaricus bisporus mushroom protects against increase in gut permeability ex vivo and up-regulates glutathione peroxidase 1 and 2 in hyperthermallyinduced oxidative stress in rats. Nutrients, 6(6), 2478-2492. doi.org/10.3390/nu6062478 
Meroni, M., Longo, M., \& Dongiovanni, P. (2019). Alcohol or Gut Microbiota: Who Is the Guilty? International Journal of Molecular Sciences, 20(18), 1-22. doi.org/10.3390/ijms20184568

Milosevic, I., Vujovic, A., Barac, A., Djelic, M., Korac, M., Spurnic, A. R., Gmizic, I., Stevanovic, O., Djordjevic, V., Lekic, N., Russo, E., \& Amedei, A. (2019). Gut-liver axis, gut microbiota and its modulation in the management of liver diseases: A review of the literature. International Journal of Molecular Sciences, 20(2), 1-16. doi.org/10.3390/ijms20020395

Navarro-Alarcon, M., \& Cabrera-Vique, C. (2008). Selenium in food and the human body: A review. Science of the Total Environment, 400(1-3), 115-141. doi.org/10.1016/j.scitotenv.2008.06.024

Nkansah, M., Donkoh, M., Akoto, O., \& Ephraim, J. (2019). Preliminary Studies on the Use of Sawdust and Peanut Shell Powder as Adsorbents for Phosphorus Removal from Water. Emerging Science Journal, 3, 33. doi.org/10.28991/esj-2019-01166

Nurmela, K., Heikkinen, V., Hokkanen, R., Joukamaa, M., Ylinen, A., Uitti, J., Mattila, A., \& Virtanen, P. (2015). Identification of alcohol abuse and transition from long-term unemployment to disability pension. Scandinavian Journal of Public Health, 43(5), 518-524. doi.org/10.1177/1403494815580149

Rayman, M. P. (2020). Selenium intake, status and health: A complex relationship. Hormones, 19(1), 9-14. doi.org/10.1007/s42000-019-00125-5

Rivière, A., Selak, M., Lantin, D., Leroy, F., \& De Vuyst, L. (2016). Bifidobacteria and butyrateproducing colon bacteria: Importance and strategies for their stimulation in the human gut. In Frontiers in Microbiology. doi.org/10.3389/fmicb.2016.00979

Roman, M., Jitaru, P., \& Barbante, C. (2014). Selenium biochemistry and its role for human health. Metallomics, 6(1), 25-54. doi.org/10.1039/c3mt00185g

Satish, K. (2003). Catechin: A TLR4 Mediated Chain Breaking Inhibitor of Signalling Cascade Involved In Endotoxin-induced Liver Injury. 5(3), 4.

Stärkel, P., \& Schnabl, B. (2016). Bidirectional Communication between Liver and Gut during Alcoholic Liver Disease. Seminars in Liver Disease, 36(4), 331-339. doi.org/10.1055/s-0036-1593882

Stärkel, P., Leclercq, S., de Timary, P., \& Schnabl, B. (2018). Intestinal dysbiosis and permeability: The yin and yang in alcohol dependence and alcoholic liver disease. Clinical Science, 132(2), 199-212. doi.org/10.1042/CS20171055
Suez, J., Korem, T., Zeevi, D., Zilberman-Schapira, G., Thaiss, C. A., Maza, O., Israeli, D., Zmora, N., Gilad, S., Weinberger, A., Kuperman, Y., Harmelin, A., Kolodkin-Gal, I., Shapiro, H., Halpern, Z., Segal, E., \& Elinav, E. (2014). Artificial sweeteners induce glucose intolerance by altering the gut microbiota. Nature. doi.org/10.1038/nature13793

Sugimoto, K., \& Takei, Y. (2017). Pathogenesis of alcoholic liver disease. Hepatology Research, 47(1), 70-79. doi.org/10.1111/hepr.12736

Sunday, N. F. (2021). Human, Earth and Future Chemometrics of Solvent Extraction of Mn (II) and $\mathrm{Fe}$ (III) Bis (salicylidene) Ethylenediamine Complexes in Acid Medium. October.

Umana, U. S., Ebong, M. S., \& Godwin, E. O. (2020). Human, Earth and Future Biomass Production from Oil Palm and Its Value Chain. 1(1), 30-38.

Wang, S. C., Chen, Y. C., Chen, S. J., Lee, C. H., \& Cheng, C. M. (2020). Alcohol addiction, gut microbiota and alcoholism treatment: A review. International Journal of Molecular Sciences, 21(17), 1-11. doi.org/10.3390/ijms21176413

Welly, R. J., Liu, T. W., Zidon, T. M., Rowles, J. L., Park, Y. M., Smith, T. N., Swanson, K. S., Padilla, J., \& Vieira-Potter, V. J. (2016). Comparison of Diet versus Exercise on Metabolic Function and Gut Microbiota in Obese Rats. Medicine and Science in Sports and Exercise. doi.org/10.1249/MSS.0000000000000964

Xu, X., Dai, M., Lao, F., Chen, F., Hu, X., Liu, Y., \& $\mathrm{Wu}$, J. (2020). Effect of glucoraphanin from broccoli seeds on lipid levels and gut microbiota in high-fat diet-fed mice. Journal of Functional Foods, 68(17), 103858. doi.org/10.1016/j.jff.2020.103858

Yan, A. W., Fouts, D. E., Brandl, J., Stärkel, P., Torralba, M., Schott, E., Tsukamoto, H., Nelson, K. E., Brenner, D. A., \& Schnabl, B. (2011a). Enteric dysbiosis associated with a mouse model of alcoholic liver disease. Hepatology. doi.org/10.1002/hep. 24018

Yan, A. W., Fouts, D. E., Brandl, J., Stärkel, P., Torralba, M., Schott, E., Tsukamoto, H., Nelson, K. E., Brenner, D. A., \& Schnabl, B. (2011b). Enteric dysbiosis associated with a mouse model of alcoholic liver disease. Hepatology, 53(1), 96-105. https://doi.org/10.1002/hep.24018

Zeng, H., \& Combs, G. F. (2008). Selenium as an anticancer nutrient: Roles in cell proliferation and tumor cell invasion. Journal of Nutritional Biochemistry, 19(1), 1-7. doi.org/10.1016/j.jnutbio.2007.02.005 
Zhai, Q., Cen, S., Li, P., Tian, F., Zhao, J., Zhang, H., \& Chen, W. (2018). Effects of Dietary Selenium Supplementation on Intestinal Barrier and Immune Responses Associated with Its Modulation of Gut Microbiota. Environmental Science and Technology Letters, 5(12), 724-730. doi.org/10.1021/acs.estlett.8b00563

Zhao, N., Guo, F. F., Xie, K. Q., \& Zeng, T. (2018). Targeting Nrf-2 is a promising intervention approach for the prevention of ethanol-induced liver disease. In Cellular and Molecular Life Sciences (Vol. 75, Issue 17, pp. 3143-3157). doi.org/10.1007/s00018-018-2852-6
Zhu, H., Zhou, Y., Qi, Y., Ji, R., Zhang, J., Qian, Z., Wu, C., Tan, J., Shao, L., \& Chen, D. (2019). Preparation and characterization of selenium enriched-: Bifidobacterium longum DD98 and its repairing effects on antibiotic-induced intestinal dysbacteriosis in mice. Food and Function, 10(8), 4975-4984. doi.org/10.1039/c9fo00960d 